\title{
Ultrasonic NDT determination of initial and final setting time of cement paste
}

\author{
Petr Misák ${ }^{1, *}$, Barbara Kucharczyková ${ }^{1}$, Dalibor Kocáb ${ }^{1}$ and Tomáš Vymazal ${ }^{1}$ \\ ${ }^{1}$ Brno University of Technology, Veveři 95, 60200 Brno, Czech Republic
}

\begin{abstract}
There are several methods for determination of the setting and early hardening in the cementitious composites. The most common method is using the Vicat apparatus. The major disadvantage of this method is that it is invasive and therefore the measurement results exhibit high variability. The paper deals with the continual monitoring of the process of setting and early hardening of a cement paste using the ultrasonic measurement. The innovative method of using the ultrasonic NDT method for determination of the setting times of cement paste is presented in the paper.
\end{abstract}

\section{Introduction}

The process of setting and hardening of binders is a complex process influenced by internal chemical and physical processes and external curing conditions. The setting times of cement (or cement paste) significantly affects not only the properties of fresh concrete (eg workability) but also of hardened concrete (shrinkage development, strength, elastic properties, etc.). It is known that the setting times of cement (binder) are different from the setting times of the final product, such as mortars or concrete [1]. Differences in setting and hardening behaviour can also be observed between the mortar produced by direct mixing of the individual components and the mortar prepared by sieving the fresh concrete [2]. There are several standardized methods for determining the setting time of binders, mortars and concrete [3-6]. All mentioned methods are based on the measurement of the penetration depth of the penetration needle (testing of binders) or resistance against penetration (testing of mortars and concrete). A major disadvantage of these methods is that they are invasive methods and the fact that one method cannot be used for all three types of cement-based materials. Besides the different dimensions of the penetration tools, the size of the specimen is also different. The most widely used and standardized method for determining the setting times of cement is using the Vicat apparatus according to EN 196-3 [3]. The setting time is considered as a time after which the needle penetrates the cement paste of normal consistency to the specific depth [3].

The aim of this paper is to present the possibilities of using the ultrasonic pulse method for continuous monitoring of the process of setting and hardening of cement paste by Vikasonic apparatus. The main advantage of this method is that it is the non-destructive and non-invasive method, as well as the ability to monitor the behaviour of all three types above mentioned cementitious materials (binders, mortars, and concrete) using the same test

* Corresponding author: petr.misak@ vutbr.cz 
configuration. A new method for evaluating the setting times based on the ultrasonic (US) pulse velocity method and the internal temperature measurement in the specimen is presented in this paper.

\section{Experimental part}

\subsection{Measuring method}

Ultrasonic apparatus Vicasonic was used for the purpose of the experiment. The principle of measurement by the Vikasonic apparatus is based on monitoring the transit time of ultrasonic pulse through the material, from which the velocity of the US wave propagation is calculated. The Vikasonic apparatus consists of two US transducers (transmitter and receiver) fixed at the top and bottom of the measuring chamber, the Vicat ring, a temperature sensor, and a data logger. The measuring base (a distance between transducers) is defined by the height of the Vicat ring. The $54 \mathrm{kHz}$ transducers are capable to transmit and receive US pulses ranging from 6 to 120 pulses per minute. During the measurement, the pulse transmitter is located on the top and the receiver on the bottom of the Vicat ring. The test configuration is shown in Fig. 1. The actual values of measured properties are shown on the display and the data are recorded digitally to a USB flash drive during the whole time of the test duration.

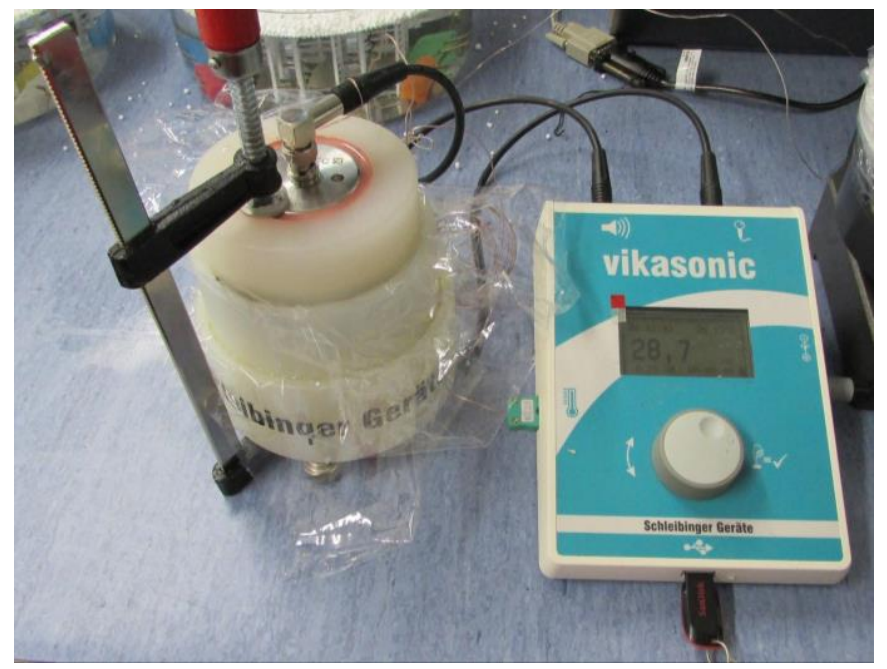

Fig. 1. Vikasonic apparatus.

Simultaneously with monitoring the US pulse transit time, the temperature inside the test specimens can be recorded using the temperature sensor inserted through the upper part of the measuring chamber. Performed experiments showed that the monitoring of the actual development of the internal temperature of the specimens is crucial for evaluating the setting times using ultrasonic measurement. The temperature curve reflects the ongoing chemical reactions of the hydration process, which are related to the early phase of the solidification process of cementitious materials. The measurement procedure includes also the calibration step, which eliminates the time losses due to the presence of the coupling agent and potential time losses related to the type of the used cables and transmitters. This calibration can be performed using the reference bar supplied with the Vikasonic apparatus performed before the start of the measurement. This calibration may be influenced by inaccuracies due to the 
filling and final closing of the measuring chamber. Therefore, the authors use another procedure for calibration of the measurement which is explained in the following paragraphs.

Fig. 2 shows the output of measurement performed on the cement paste of normal consistency prepared according to the EN 196-3 [3]. The cement paste of normal consistency was prepared using CEM I 42.5 R. The results are discussed in the following sections. In this section, just a general procedure used for data evaluation is described in detail. The same procedure is used also for the evaluation of measurement performed for other cement paste prepared from CEM I $52.5 \mathrm{R}$ cement.

The data of US pulse velocity calculated based on a real measured US pulse transit time and real measured temperature curve were smoothed by using a cubic spline for the purpose of later data processing. The authors use supplemental US device PUNDIT to perform the calibration of the measurement. One of the motivations for this approach is the frequency of ultrasonic transmitters. The Vikasonic apparatus is equipped with the transmitters of the frequency of $54 \mathrm{kHz}$ which is a frequency suitable for determining the US pulse transit time of the fresh cement paste. However, the suitability of these transmitters decreases during setting and hardening of the material. With respect to the length of the measuring base (height of the Vicat ring is $40 \mathrm{~mm}$ ), it can be stated that transmitters with a higher frequency are more suitable for measurement after reaching the setting time and during later hardening. Therefore, the authors recommend the following procedure. Immediately after the Vikasonic measurement is completed, the US pulse transit time of the already hardened specimen using the PUNDIT instrument or another ultrasonic instrument with a transmitter frequency of at least $150 \mathrm{kHz}$ has to be determined. Subsequently, the last measured value of US pulse transit time recorded by the Vikasonic apparatus is subtracted from the US pulse transit time determined using the PUNDIT instrument. This deviation is then subtracted from all values of the US pulse transit time recorded by the Vikasonic apparatus. The above-described calibration procedure assumes that the value of the deviation is constant during the whole time of performed measurement, which may not be true because of the changes in the properties of the investigated material during the solidification process. However, it seems that this approach appears to be the most suitable technique for calibration of measurement performed by the Vikasonic apparatus.

The procedure for determining the setting times of the cement paste of normal consistency by Vikasonic apparatus is based on the determination of the specific points on the curve of internal temperature and US pulse velocity recorded simultaneously during solidification of the cement paste. The evaluation is based on the transfer of specific points of the temperature curve to the US pulse velocity curve (see Fig. 3 ). These specific points, which can be found on the temperature curve during the first 24 hours of setting and hardening, are minimum and maximum temperature, and two inflection points, ie points with zero second derivative, in which the temperature curve changes from convex to a concave shape. Smoothing the data of the recorded temperature curve with cubic splines is beneficial for finding these points. The points transferred to the US pulse velocity curve have the same $x$ coordinate as for the temperature curve (see Fig. 3).

Evaluation of initial setting time (IST) and final setting time (FST) of cement paste of normal consistency is based on searching for intersections of tangent lines and the straight line connecting the points of inflection, see in Fig. 3. The results of the evaluation are shown in Fig. 4. It is shown that the IST is determined as the $\mathrm{x}$ coordinate of the point that results from the intersection of the tangent line to the curve of US pulse velocity through the transferred minimum temperature point and the line connecting transferred inflection points 1 and 2. The FST is then determined as the intersection of the tangent line through the transferred minimum temperature point and the tangent line through the transferred inflection point 2. The fitting of the measured data with the cubic spline simplifies the determination of these tangents. The above-described process of evaluation can be fully automated with 
appropriate software processing. In the next sections of the paper, there is shown the evaluation of the performed experiment aimed at the comparison of the setting times obtained from the measurements using the Vikasonic apparatus and the standardized test using the Vicat apparatus performed according to the EN 196-3 [3].

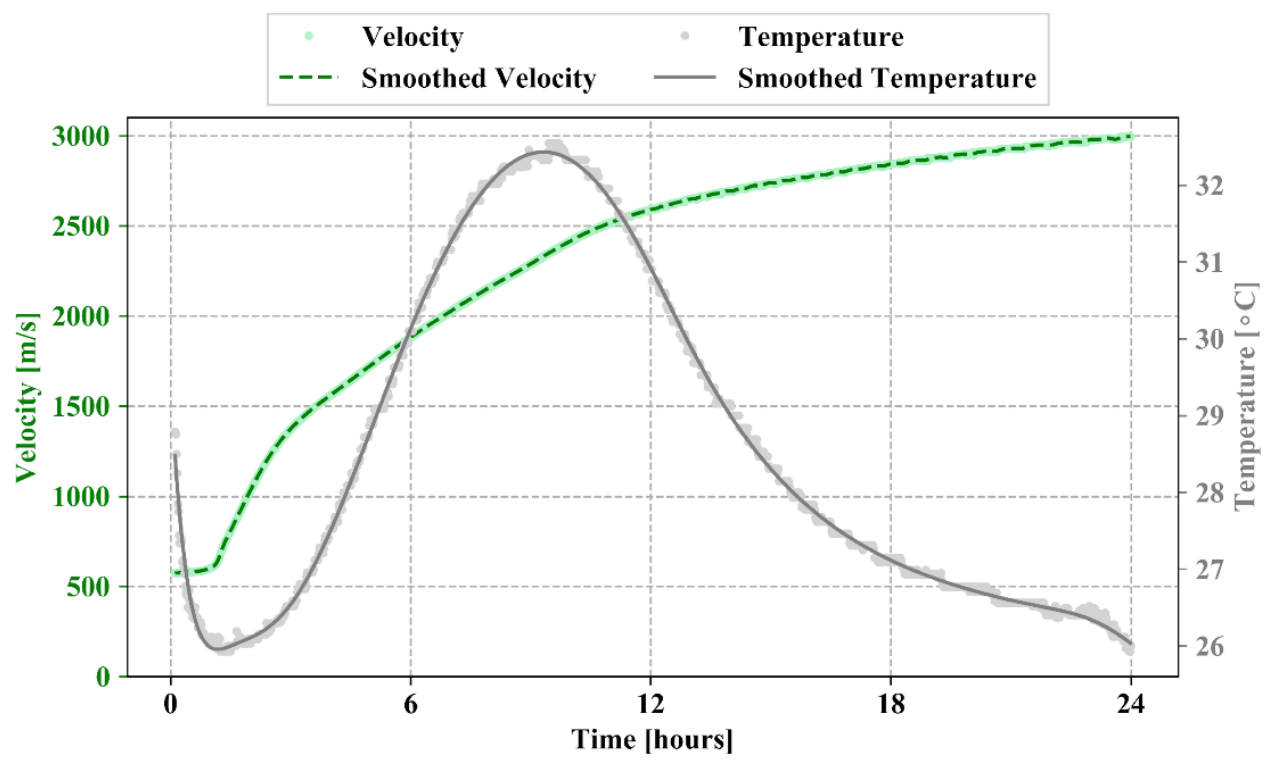

Fig. 2. Vikasonic measurement - CEM I 42.5 R.

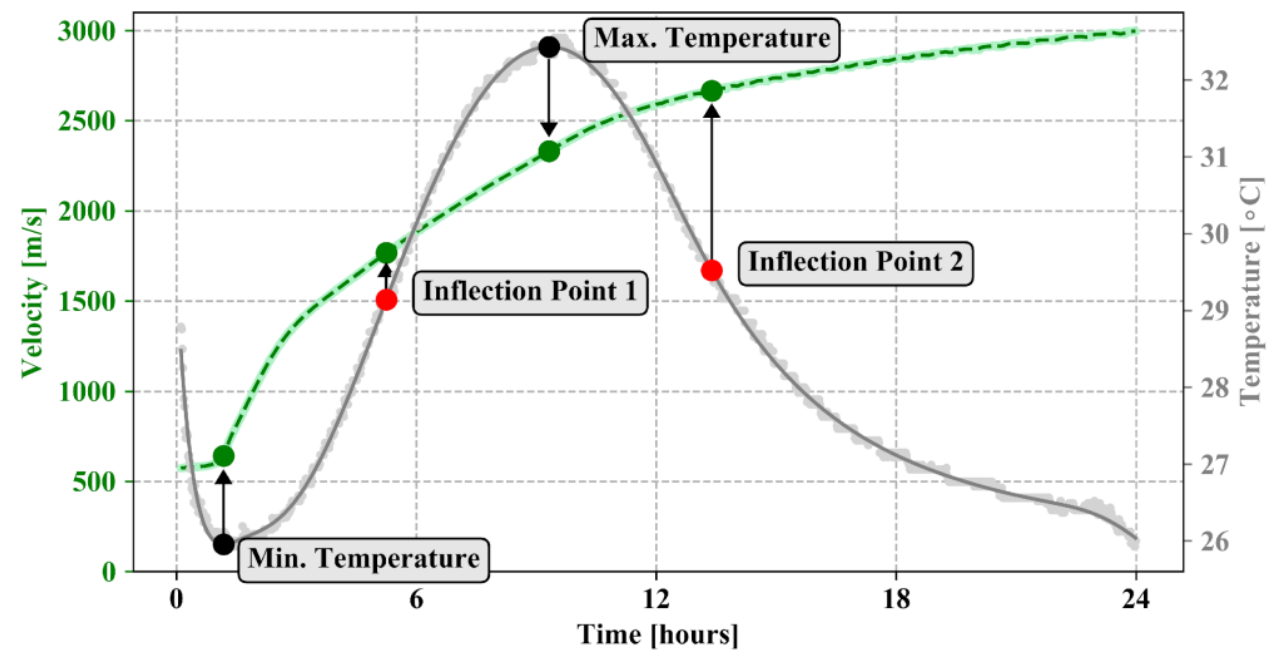

Fig. 3. Important points on the temperature curve and their transfer-CEM I $42.5 \mathrm{R}$. 


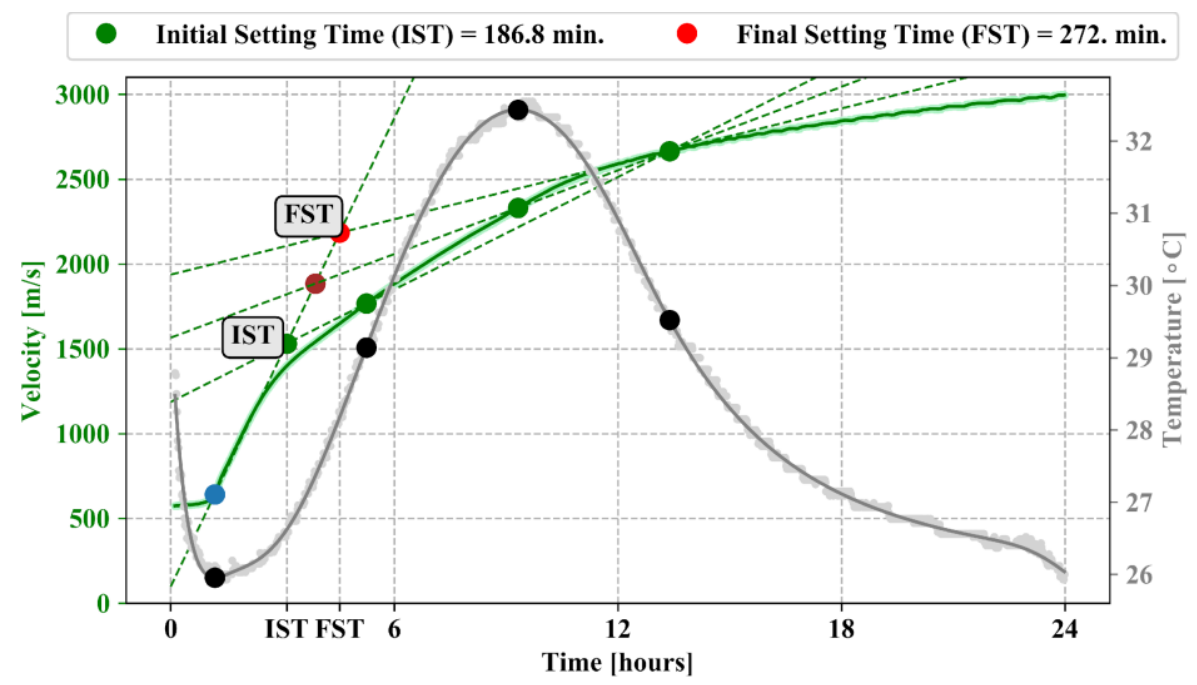

Fig. 4. Evaluation of initial setting time (IST) and final setting time (FST) - CEM I 42.5 R.

The advantages and disadvantages of the standardized and well-known test method intended for the determination of setting times of the cement paste of normal consistency according to the EN 196-3 [3] have already been discussed in the introduction. A wider discussion about this method can be found eg in the article [7]. The origins of this method can be found in the $19^{\text {th }}$ century when the cement was for the first time used as a building material. The straightforwardness and simplicity of this method cannot be denied. However, it seems that the measurement procedure is not adequate to meet the demands posed to the materials testing in the $21^{\text {st }}$ century, especially with regard to the high variability of its results. This variability may be related to the invasive nature of this method. The resulting value of IST and FST can be influenced by local inhomogeneities in the specimens. For this and other reasons, it seems appropriate to look for other methods that are able to give more details about the changes in the internal structure of materials during the solidification process. Based on the performed experiment it can be stated that the ultrasonic pulse method can be used as a supplement or a substitute for the EN 196-3 method [3].

\subsection{Experiment}

The experiment shows the comparison of the IST and FST values obtained from the measurement using two different testing methods - ultrasonic and Vicat test. Two types of cement were tested - CEM I 42.5 R and CEM I 52.5 R. The cement was produced at the Mokrá cement plant (Czech Republic). The outputs of measurement performed for CEM I 42.5 R cement using the Vikasonic apparatus is shown in Fig. 1 - 4. In Fig. 5 there is shown a record and evaluation of a Vicat apparatus test for CEM I $42.5 \mathrm{R}$ cement. Because the standard [3] defines IST as the time when the distance of the penetration needle from the bottom of Vicat ring is in the range of $6 \pm 3 \mathrm{~mm}$, the IST is in Fig. 5 shown as a time interval. The real measured data were fitted with a cubic spline for the purpose of measurement evaluation. The values of the setting times were determined from the fitted curve, not directly from the real measured data. The same procedure of the data obtained from Vicat test was used for the evaluation of measurement performed for CEM I 52.5 R cement. 


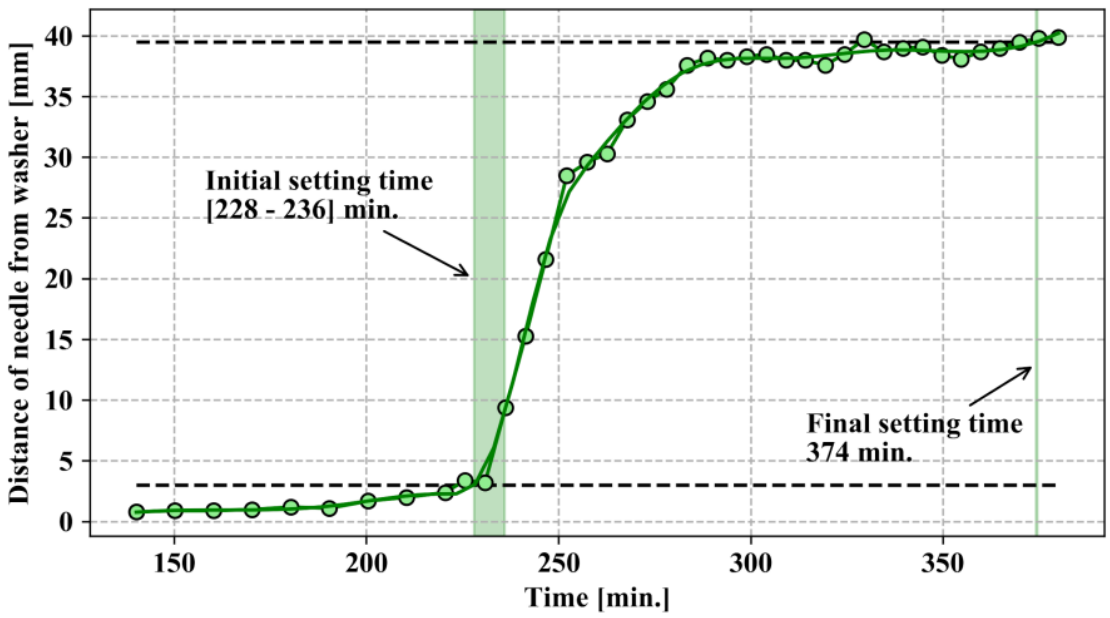

Fig. 5. Vicat method - CEM I 42.5 R.

\subsection{Results and discussion}

Tab. 1 shows a comparison of the obtained test results together with the data given in the technical sheet of a particular type of cement. In the case of CEM I $42.5 \mathrm{R}$ cement, the value of IST obtained using the Vikasonic apparatus corresponds very well to the IST given in the technical sheet of cement. On the other hand, the testing method using the Vicat apparatus shows significant differences. It should be noted that the data given in the technical sheet of cement consists of an evaluation of a large set of measurements, while in the case of the presented experiment only one measurement was performed that could be influenced by a systematic error. In the case of CEM I $52.5 \mathrm{R}$, the differences between the obtained data are within reasonably assigned measurement uncertainty.

Table 1. Comparison of test results and data in the technical data sheet of cement producer.

\begin{tabular}{|l|l|c|c|c|}
\hline Cement & Characteristics & $\begin{array}{c}\text { Technical } \\
\text { data sheet } \\
\text { [min.] }\end{array}$ & $\begin{array}{c}\text { Vicat method } \\
\text { [min.] }\end{array}$ & $\begin{array}{c}\text { Vikasonic } \\
\text { method } \\
\text { [min.] }\end{array}$ \\
\hline \multirow{2}{*}{ CEM I 42.5 R } & Initial setting time (IST) & 183 & $228-236$ & 187 \\
\cline { 2 - 5 } & Final setting time (FST) & 242 & 374 & 272 \\
\hline \multirow{2}{*}{ CEM I 52.5 R } & Initial setting time (IST) & 143 & $124-144$ & 157 \\
\cline { 2 - 5 } & Final setting time (FST) & 196 & 222 & 230 \\
\hline
\end{tabular}

The manufacturer of Vikasonic apparatus states that the correlation between both herein mentioned methods is different for different materials, i.e. types of cement [8]. It means that for each cement it is possible to make a relationship between the results of US tests and the penetration distance determined according to [3]. Based on this relationship the setting times can be determined. However, searching for such relationships can be influenced by several factors. One of them is the different sensitivity of both methods. While the US method is able to reflect the changes in material structure almost immediately after mixing the cement with water, the penetration method [3] starts to show significant changes around the time of the initial setting time. In addition, using the Vikasonic apparatus, the development of material 
maturation even after the final setting time can be monitored, which is almost impossible by the penetration method according to [3]. As the experimental data shows, the searching of the relationship between these methods may not lead to a satisfactory goal, i.e. to find the setting times of the cement paste. In Fig. 4 it is shown that the curve of the US pulse velocity between IST and FST points is almost linear. Therefore, it is obvious that by combining the outputs of both methods the curve with a similar shape as shown in Fig. 5 can be obtained. Based on authors' experience with the measurement using Vikasonic apparatus, it seems to be necessary to use simultaneous measurement of the temperature inside the material for the purpose of evaluation of setting times which enables to evaluate the setting times directly from the US measurement, as described in this article, without need for finding the relationship between the penetration method. Therefore, finding a relationship to determine setting times is not necessary. The principle of utilizing the development of the internal temperature of the material during ageing to determine setting times is also applied in the ASTM recommendations [9].

\section{Conclusion}

The aim of this paper was to show the possibilities of using the ultrasonic pulse method to determine the initial and final setting time of cement paste. The main advantage of ultrasonic measurement is that it is the non-invasive method. This should result in reduced variability of test results. Based on the experiment, the ultrasonic pulse method appears to be useful for investigating the initial stages of setting and hardening process of the cement paste. The dimensions of the measurement chamber enable to use of the Vikasonic apparatus for testing other common types of cement composites such as mortars and concrete with a maximum aggregate grain size of $8 \mathrm{~mm}$ using the same test configuration.

This paper has been written as a part of project No. GA17-14302S, supported by the GAČR - Czech Science Foundation.

\section{References}

1. A. M. Neville, Properties of concrete (5th ed. New York: Pearson, 2011).

2. C. H. Lee, K. C. Hover, A. Lee. Journal of Testing and Evaluation. 2015, 43(4).

3. EN 196-3. Methods of testing cement - Part 3: Determination of setting time and soundness (2017).

4. ČSN 73 1332. Determination of concrete setting (Praha, CEN, 1986).

5. ASTM C403 / C403M - 16: Standard Test Method for Concrete Mixtures by Penetration Resistance (ASTM International, West Conshohocken, PA, 2016).

6. ASTM C191-13. Standard Test Methods for Time-Based Hydraulic Cement by Vicat Needle (ASTM International, West Conshohocken, PA, 2013).

7. H. Sleiman, A. Perrot, S. Amziane. Cement and Concrete Research. 2010, 40(5), p. 681686.

8. Vikasonic - Measuring the Early Setting and Hardening with Ultrasonic Waves [online]. September 16, 2019. Available from: http://www.schleibinger.com/cmsimple/en/?Setting_and_Maturity:Ultrasonic_Setting_ Measurement.

9. ASTM C1679-14. Standard Practice for Measuring Hydration Kinetics of Hydraulic Cementitious Mixtures Using Isothermal Calorimetry (ASTM International, West Conshohocken, PA, 2014). 